Thomas Prantner*

\title{
Die zeit- und kulturhistorischen Videoarchive der ORF-TVthek
}

https://doi.org/10.1515/bfp-2020-2033

Zusammenfassung: Die ORF-TVthek stellt neben tagesaktuellem TV-Programm inzwischen 36 themenbezogene zeit- und kulturhistorische Videoarchive mit insgesamt 3500 Videos im Rahmen der Aktion „ORF-TVthek goes school“ auch speziell für die Nutzung im Unterricht bereit. Wertvolle Inhalte des Multimedialen ORF-Archivs dienen dabei dazu, wichtige Ereignisse und Entwicklungen öffentlich zugänglich zu machen, in multimedialer Form zu dokumentieren und das Verständnis für Vergangenheit und Gegenwart im Sinne des öffentlich-rechtlichen Bildungsauftrags zu fördern, v. a. bei der jungen Zielgruppe.

Schlüsselwörter: Videoarchive; ORF-TVthek; Video-Streaming-Plattform; Mediathek

\section{The ORF-TVthek's Video Archives on Contemporary and Cultural History}

Abstract: In addition to daily TV programs, the video platform ORF-TVthek provides 36 topic-related historical and cultural video archives with a total of 3,500 videos, especially for use in schools as part of the "ORF-TVthek goes school" project. Valuable content of the multimedia ORF archive is used to document important events and developments in a publicly accessible manner in multimedia form and to promote the understanding of the past and the present according to ORF's public education mandate, especially among the young target group.

Keywords: Video archives, ORF-TVthek, video streaming platform; media library

Die Nutzung der neuen digitalen Medienplattformen für die öffentlich-rechtlichen Programminhalte des ORF (Österreichischer Rundfunk) ist zentrale Zielsetzung der Multimedia-Strategie des Unternehmens. Mit der 2009 gegründeten Videoplattform ORF-TVthek ist es dem ORF gelungen, einen modernen digitalen TV-Zusatzdienst zu schaffen, der das aktuelle ORF-TV-Programm auf allen wichtigen medialen und technologischen Plattformen bereitstellt und damit das steigende Bedürfnis des Publikums

*Kontaktperson: Thomas Prantner, thomas.prantner@orf.at nach zeit- und ortsunabhängig nutzbaren österreichischen Programmen optimal abdeckt.

Gleichzeitig dient die ORF-TVthek mit ihren dutzenden zeit- und kulturhistorischen Videoarchiven als Plattform für die themenbezogene Online-Bereitstellung von hochwertigen ORF-Contents der vergangenen Jahrzehnte.

\section{Rechtliche Rahmenbedingungen}

Die Onlineaktivitäten des öffentlich-rechtlichen Rundfunks ORF sind im ORF-Gesetz geregelt. Dieses erlaubt dem ORF, Eigen-, Auftrags- und Koproduktionen im Rahmen von Abrufdiensten für sieben Tage nach der TV-Ausstrahlung (7-Days-Catch-Up), im Fall von Premium-Sportarten für 24 Stunden nach der TV-Ausstrahlung online als Video-on-Demand-Streams bereitzustellen.

Eine wesentliche Ausnahme von dieser Einschränkung sind Archive mit zeit- und kulturgeschichtlichen Inhalten. Diese dürfen auch zeitlich unbefristet zum Abruf bereitgestellt werden. ${ }^{1}$ Diese Ausnahmeregelung wird vom ORF dazu genutzt, zahlreiche Archiv-Inhalte themenbezogenen via Internet einer breiten Öffentlichkeit erneut bekannt bzw. langfristig verfügbar zu machen.

\section{Das tagesaktuelle ORF-TVthek-Angebot}

Der ORF stellt, soweit gesetzlich und lizenzrechtlich möglich, sein tagesaktuelles TV-Programm als Video-on-Demand auf der ORF-TVthek ${ }^{2}$ bereit. Die Sendungen bleiben nach der TV-Ausstrahlung für sieben Tage on Demand verfügbar. Etwa die Hälfte des Angebots muss mit Geoprotection-Beschränkung auf Österreich ${ }^{3}$ bereitgestellt werden.

Auf der ORF-TVthek wird die Philosophie einer möglichst bequemen und schnellen Auffindbarkeit und Abruf-

1 Siehe dazu ORF-Gesetz unter https://www.ris.bka.gv.at/GeltendeF assung.wxe?Abfrage=Bundesnormen\&Gesetzesnummer=10000785. 2 https://TVthek.ORF.at.

3 Stand 2019, interne Auswertung. 


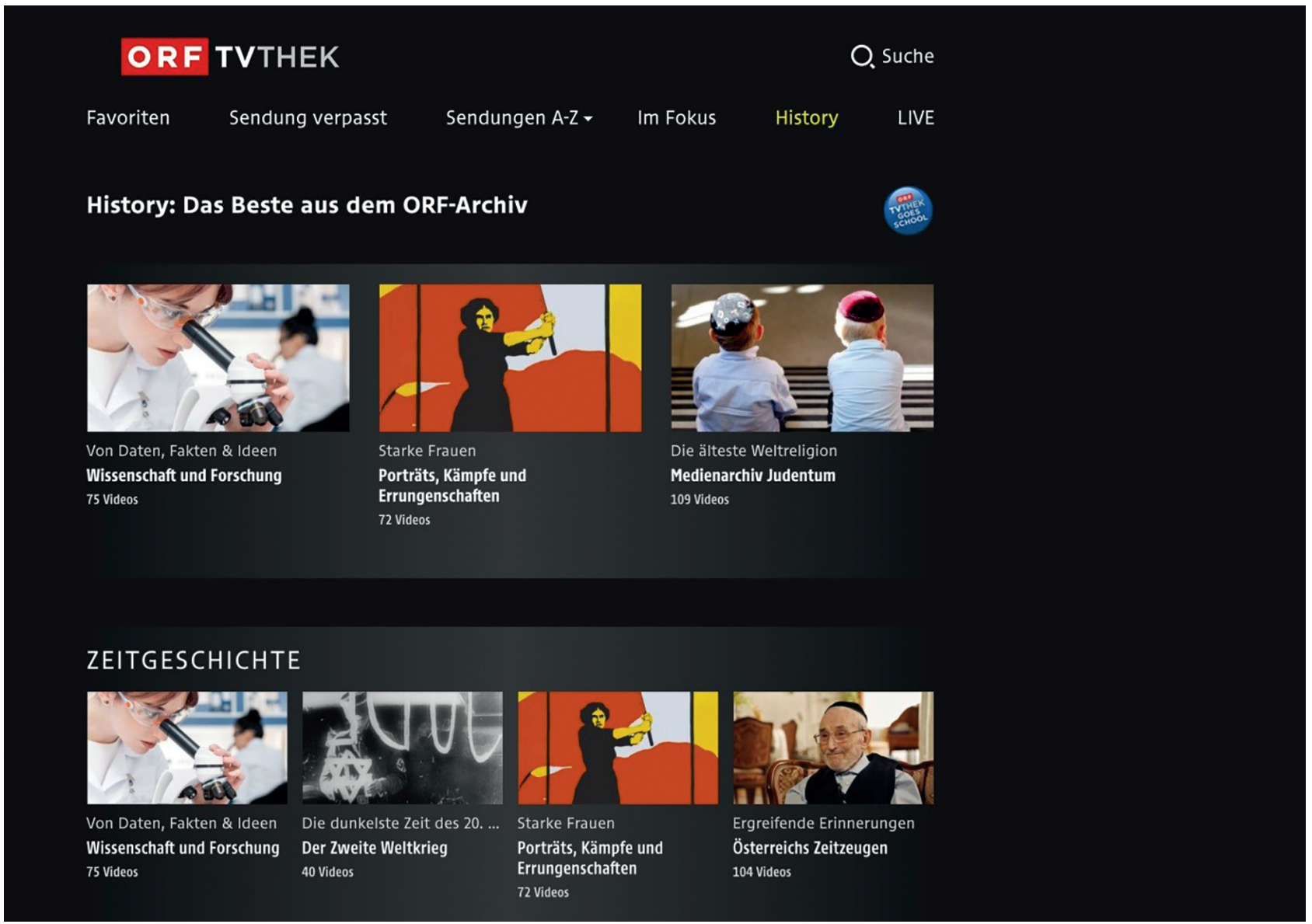

Abb. 1: Die zeit- und kulturhistorischen Videoarchive der ORF-TVthek

barkeit gewünschter Einzelinhalte umgesetzt, indem Sendungen nicht nur als Ganzes in einem Video-on-DemandFile, sondern dort wo sinnvoll auch gegliedert in Einzelbeiträge (zum Beispiel bei Nachrichtensendungen) online sind.

Das tagesaktuelle Angebot umfasst Sendungen der Sender ORF 1 und ORF 2 sowie der Spartenkanäle ORF III (Kultur und Information) und ORF SPORT+. Abrufbar sind alle Eigen-, Auftrags- und Koproduktionen des ORF, für die der ORF die entsprechenden Online-Lizenzrechte hält, sowie diverse hochwertige Kaufproduktionen. Alle Genres von Magazin-, Informations-, Regional-, Kultur-, Sport-, Service-, Religions-, Dokumentations- sowie Unterhaltungssendungen bis zu Sendungen des Kinderprogramms, Filme und Serien sind vertreten.

Das tagesaktuelle Livestream-Angebot umfasste im Jahr 2019 insgesamt bereits 76 Prozent der vier ORF-TVProgramme, davon 65 Prozent mit Geoprotection, ${ }^{4}$ d.h., der Abruf dieser Inhalte ist auf Österreich begrenzt. Hinzu

4 Interne Auswertung. kommt das LIVE-SPEZIAL-Service der ORF-TVthek, welches zusätzlich und ergänzend zur tagesaktuellen TV-Berichterstattung, vom ORF oder der APA (Austria Presse Agentur) produzierte Live-Videoaufnahmen als unkommentierte Zusatz-Livestreams von Pressekonferenzen, Reden und Veranstaltungen aus Politik, Wirtschaft, Kultur, Chronik und Sport als Additional Content zur Verfügung stellt.

\section{Bereitstellung von Archiv-Inhalten}

Bereits seit 2011 nutzt der ORF darüber hinaus die Möglichkeit zur Bereitstellung zeit- und kulturhistorischer Videoarchive, die auf Basis von Sendungen des ORF-Archivs einen multimedialen Rückblick auf historische und kulturelle Ereignisse geben. Im Rahmen dieser Videoarchive können - themenbezogen - Sendungen und Beiträge des ORF-Fernsehens aus den vergangenen Jahrzehnten für das Publikum langfristig und einfach online verfügbar ge- 
macht werden. Die Archive sind als einziger Inhalt der Videoplattform des ORF ohne zeitliche Befristung verfügbar.

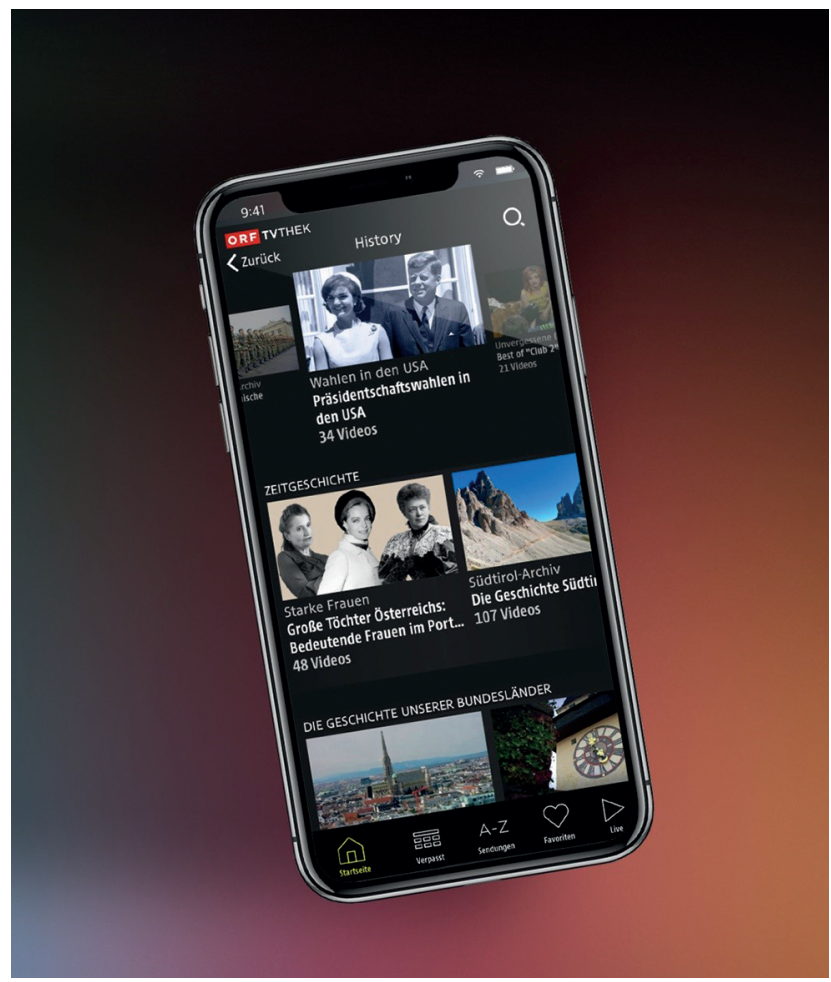

Abb. 2: ORF-TVthek und ihre Videoarchive auch für die mobile Nutzung verfügbar

Die Funktion des ORF als multimediales Gedächtnis Österreichs und als Vermittler historischer Ereignisse und Entwicklungen wird mit den ORF-TVthek-Archiven um eine wichtige Facette reicher. Vor allem kann damit eine wertvolle und einzigartige, bereits in Form von professionell gestalteten Sendungen und Beiträgen vorliegende Quellensammlung auch der besonders online-affinen jungen Zielgruppe nähergebracht werden. Die ORF-TVthek-Archive dienen dabei nicht nur der Dokumentation von Vergangenheit und Gegenwart in audiovisueller Form, sondern tragen durch die potenzielle Erweiterung und Vertiefung der allgemeinen Kenntnisse des Publikums zu diversen gesellschaftspolitisch relevanten Themen zum besseren Verständnis der Gegenwart bei.

Der ORF ergänzt durch diese Nutzung der Technologien des digitalen Zeitalters außerdem sein Portfolio und kann seinem Publikum einen wichtigen öffentlich-rechtlichen Mehrwert anbieten. Gleichzeitig wird die Erfüllung des Bildungsauftrags durch zeitgemäße, moderne und multimediale Wissensvermittlung maßgeblich unterstützt.

\section{ORF-TVthek goes school}

Im Rahmen der Aktion „ORF-TVthek goes school“ wurde im Frühjahr 2014 außerdem mit dem Launch von speziell für die Nutzung im Unterricht geeigneten Online-Videoarchiven zu Themen aus den Bereichen Geschichte, Kultur, Politik, Religion und Chronik gestartet. Im Rahmen dieser Aktion sollen die Videoarchive vor allem Lehrer/innen und Schüler/innen ansprechen.

Sie sind speziell als multimediales Bildungsangebot für den Unterricht an Schulen und weiteren Bildungseinrichtungen geeignet. Vom ORF gestaltete Fernsehsendungen und -beiträge zu zeit- und kulturhistorischen Events und Entwicklungen können somit dabei behilflich sein, v. a. Jugendlichen Bildungsinhalte mittels moderner Kommunikationsmedien näherzubringen.

\section{6 zeit- und kulturhistorische Videoarchive}

Insgesamt stehen derzeit bereits 36 zeit- und kulturhistorische Videoarchive und rund 3500 Videos (Sendungen und Beiträge) von herausragenden TV-Produktionen der vergangenen Jahrzehnte aus dem ORF-Archiv auf der ORFTVthek zur Verfügung. Die Videoarchive sind unter http s://TVthek.ORF.at/history nicht nur österreichweit uneingeschränkt und unbefristet verfügbar, sondern soweit möglich auch weltweit zugänglich. ${ }^{5}$ Alle Angebote der ORFTVthek, auch die Videoarchive, sind kostenfrei und ohne Registrierung nutzbar.

\section{Breite Themenpalette}

Das Gesamtangebot an Videoarchiven umspannt eine bunt gefächerte Palette an Themen in den Kategorien Zeitgeschichte, Geschichte der österreichischen Bundesländer, Politik und Wirtschaft, Religion sowie Fernseh- und Mediengeschichte, mit denen das Interesse der User/innen geweckt werden soll.

Die einzelnen Themen reichen dabei von der „Geschichte der EU“ oder der „Politischen Geschichte der Zweiten Republik“, über das „Schicksalsjahr 1938“, „Starke Frauen: Porträts, Kämpfe und Errungenschaften“ oder einem Zeitzeugen-Archiv bis zur Geschichte der Volks-

5 Ausnahme sind Beiträge, für die keine entsprechenden Lizenz- und Urheberrechte vorliegen. 


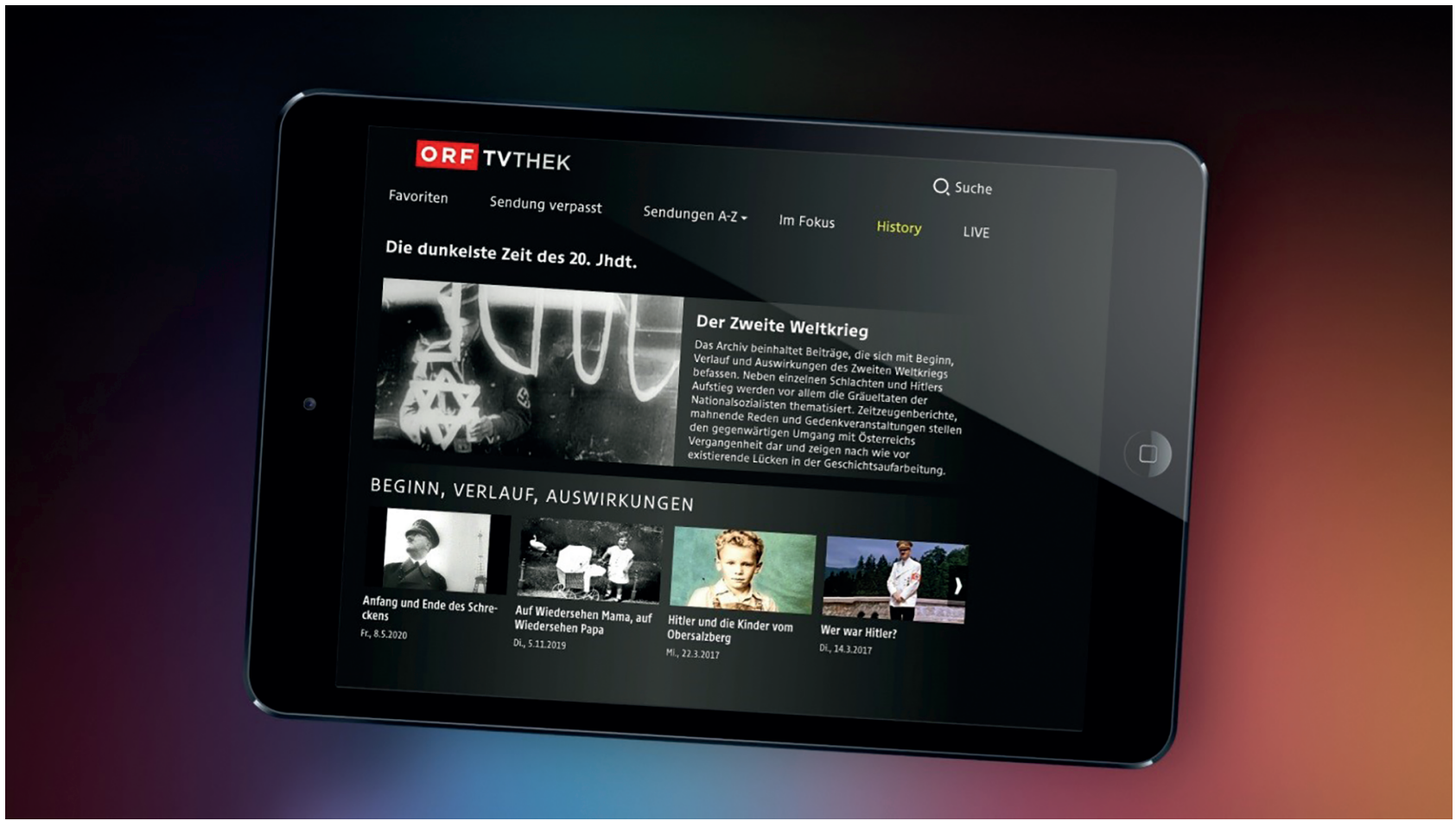

Abb. 3: Startseite des ORF-TVthek-Videoarchivs zum Thema Zweiter Weltkrieg

gruppen, oder den Highlights aus 60 Jahren ORF-TV-Geschichte.

Die neuesten Archive befassen sich mit den Themenkomplexen Nachhaltigkeit und Klimaschutz sowie der Coronavirus-Krise (beide Launch September 2020), „Wissenschaft und Forschung“ (Launch Juni 2020), „Zweiter Weltkrieg“ (Launch August 2019) und „Volksgruppen in Österreich“ (Launch März 2019).

Laufend werden sowohl die bestehenden Videoarchive erweitert - so zum Beispiel im Juni 2020 das „Medienarchiv Judentum“ - als auch neue bereitgestellt.

\section{Themenauswahl und Workflow}

Die Themenauswahl erfolgt zum einen anlassbezogen, wenn sich beispielsweise die Gründung der Zweiten Republik, oder das Ende des Zweiten Weltkrieges jährt. So wurde etwa das neue Videoarchiv zum Themenkomplex Nachhaltigkeit und Klimaschutz rund um den entsprechenden Mutter-Erde-Programmschwerpunkt des ORF bzw. den Austrian World Summit im September 2020 gelauncht.

Andererseits verfügt das ORF-Archiv über mannigfaltige historische Beiträge, Sendungen, Livemitschnitte, Interviews etc., die, entsprechend zusammengestellt und spannend aufbereitet, dabei helfen können, das Interesse des
Publikums und insbesondere der Jugend an speziellen Aspekten der Geschichte und Kultur Österreichs und anderer Staaten zu wecken - ob etwa an besonderen Traditionen und historischen Meilensteinen des eigenen Bundeslands oder an der Entstehung der EU.

Die einzelnen Videoarchive werden dabei in enger Zusammenarbeit von TVthek-Redaktion, Multimedialem Archiv und Rechtemanagement konzipiert und gestaltet. Weiters werden je nach thematischem Bezug andere ORFBereiche oder Fachabteilungen (von den Landesstudios bis zur Religionsabteilung) bei der Planung hinzugezogen.

Häufig können auch andere Institutionen - zum Beispiel das Jüdische Museum Wien bei der Erweiterung des Medienarchivs Judentum, dafür gewonnen werden, ihre Expertise im Rahmen von Kooperationen in das Projekt mit einzubringen.

\section{Auflistung der ORF-TVthek-Videoarchive ${ }^{6}$}

\author{
Zeitgeschichte \\ - Wissenschaft und Forschung
}

6 Stand September 2020. 
- Der Zweite Weltkrieg

- Starke Frauen: Porträts, Kämpfe und Errungenschaften

- Österreichs Zeitzeugen

- Die Geschichte Südtirols

- Das Schicksalsjahr 1938

- Die politische Geschichte der Zweiten Republik

- 100 Jahre Erster Weltkrieg

- Der Fall des Eisernen Vorhangs

- Die Geschichte der Ersten Republik

- Kampf dem Klimawandel - Nachhaltigkeit und Umweltschutz

- Coronavirus-Pandemie

Die Geschichte der österreichischen Bundesländer

- Die Geschichte Wiens

- Die Geschichte Niederösterreichs

- Die Geschichte des Burgenlands

- Die Geschichte Oberösterreichs

- Die Geschichte der Steiermark

- Die Geschichte Tirols

- Die Geschichte Vorarlbergs

- Die Geschichte Salzburgs

- Die Geschichte Kärntens

- Volksgruppen in Österreich

Politik und Wirtschaft

- Das Österreichische Bundesheer

- Bundestagswahlen in Deutschland

- Bundespräsidentenwahlen in Österreich

- Nationalratswahlen in Österreich

- Causa Hypo

- Die Geschichte der EU

- Präsidentschaftswahlen in den USA
Religion

- Medienarchiv Judentum

- Medienarchiv Christentum

Fernseh- und Mediengeschichte

- Bewegende Momente aus „Bundesland heute“

- 60 Jahre TV: Historische Rückblicke

- Best of ZiB 2-Interviews

- Best of Club 2

- Österreich beim Song Contest

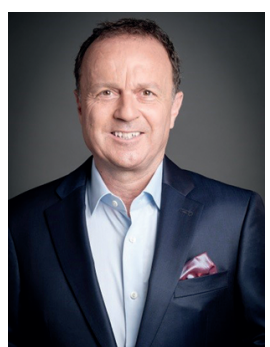

Thomas Prantner

Stv. ORF-Direktor für Technik

Online und neue Medien

Würzburggasse 30

A-1136 Wien

Österreich

thomas.prantner@orf.at 